\title{
La violencia feminicida: abordaje desde una geografía feminista
}

\section{Femicide Violence: An Approach from a Feminist Geography}

Angélica Lucía Damián BernaL Facultad de Filosofía y Letras, Universidad Nacional Autónoma de México

México

Verónica IBARRA GARCÍA Facultad de Filosofía y Letras, Universidad Nacional Autónoma de México

México

\section{Resumen}

Este trabajo aborda la violencia feminicida como un proceso social enraizado en el patriarcado y el capitalismo salvaje que menosprecia, invisibiliza y ataca a las mujeres. Identificamos los elementos de la conexión del sistema legal y la contienda social. De la geografía feminista crítica a la posibilidad de cambio y el compromiso de transformar condiciones injustas, plantea el espacio de género como una producción social de alta complejidad. Aquí vemos con precisión cómo el movimiento feminista, conformado por activistas, agrupaciones y familias de mujeres víctimas de violencia, incluyendo la academia y diversas corrientes feministas presentes en el ámbito político, ha impulsado cambios a nivel jurídico. Sin embargo, estas transformaciones han desatado resistencias en grupos conservadores. En los Censos Nacionales de 2018 se elaboró un estudio empírico con base en estadísticas de la procuración e impartición de justicia. A partir de estos datos de las entidades estatales se elaboraron mapas nacionales que permiten dar cuenta de la resistencia a clasificar los delitos contra las mujeres como feminicidios. Considerando el marco teórico basado en la tríada metodológica constitutiva del espacio, nos referimos a la creación de espacios libres de violencia, la complejidad que conlleva y la resistencia que se debe superar a través del movimiento feminista y la transformación de los marcos legales.

Palabras clave: violencia feminicida, geografía feminista, espacio feminicida en México

\begin{abstract}
This work approaches femicide violence as a social process rooted in the patriarchy and the savage capitalism that belittle, make invisible, and attack women. We identified
\end{abstract}




\section{$32 \square$ LA VIOLENCIA FEMINICIDA: ABORDAJE DESDE UNA GEOGRAFÍA FEMINISTA}

the connection between the legal system and social contest. From critical feminist geography to the possibility of change and commitment to transform unjust conditions, it poses gendered space as an overly complicated social production. Here we accurately see how the feminist movement, made up of activists, groups, and families of women victims of violence, including the feminist academy and diverse feminist strands present in the political sphere, has promoted changes at the legal level. Nevertheless, these transformations have unleashed resistance in conservative groups. An empirical study based on justice procurement and Justice administration was developed during the National Censuses of 2018. Based on these data, national maps were prepared by state entities that allow accounting for resistance to classifying crimes against women as femicides. We contribute to the theoretical framework based on the methodological triad constitutive of space. We refer to creating spaces free of violence, the complexity that it entails, and the resistance that must be overcome through the feminist movement and the transformation of legal frameworks.

Keywords: femicide violence, feminist geography, femicide space in Mexico

\section{Introducción}

El presente trabajo se aborda desde una geografía feminista y asume que tanto el espacio como la violencia contra las mujeres se producen socialmente a través de las acciones y omisiones jurídico-políticas, sociales, culturales y económicas. Por tanto, podría erradicarse con distintas acciones en esos ámbitos articulados en las distintas escalas. La geógrafa Doreen Massey (2007) considera al espacio social producto de las acciones, relaciones y prácticas sociales y por consiguiente abierto a la política, evocando que, si lo producimos, igualmente podemos transformarlo (s. p.). En concordancia con nuestra autora, consideramos que la manifestación espacial de la violencia contra las mujeres se produce tanto por las acciones de agresión contra las mismas, como por las inacciones preventivas, de atención, acceso a la justicia y la falta de respuesta para su erradicación, sostenidas por la impunidad del estado que tienen su correlato en una sociedad patriarcal en la que los medios masivos de comunicación (particularmente televisión, prensa y radio) y los elementos culturales como la música, la literatura, el teatro, el cine, la pintura, todas las artes en general, naturalizaban o aún naturalizan dicha violencia. También podemos incluir todas las actividades deportivas y recreativas en donde a las mujeres se les cosifica, se les estereotipa y se les margina o agrede, acorde al sistema patriarcal hegemónico.

Por ello, la violencia contra las mujeres se tiene que visibilizar, nombrar, identificar, detectar y sancionar para, así, reconocer que es un problema estructural y público que se debe frenar. Desde la geografía feminista hay un posicionamiento político, reconociendo que en las manifestaciones de violencia contra las mujeres intermedian relaciones de poder patriarcal y una ideología misógina en las que intervienen varios 
factores. Trasciende el acto de agresión en sí mismo que se sostiene por las escalas articuladas en las que predomina una estructura de abuso, discriminación y subordinación en contra de las mujeres, que se configura por un elaborado entramado social, cultural, jurídico y económico que protege, respalda y encubre al agresor o agresores. El sistema judicial, la sociedad, los medios de comunicación imbuidos en una ideología patriarcal, al perdonar y solapar a los agresores, producen espacios patriarcales caracterizados por la impunidad. Los hechos violentos no sancionados por el sistema institucional del estado producen impunidad, con lo cual el estado se convierte en responsable y cómplice de los hechos (Damián, 2016: 31). En ese sentido, la violencia contra las mujeres se ejerce de manera estructural, ya que está ensamblada por elementos políticos, culturales, sociales, económicos y educativos en los que se permea una visión misógina que invisibiliza, menosprecia y justifica la agresión a las mujeres en todos los espacios. El caso más extremo es la violencia feminicida, aquella "violencia de género contra las mujeres, producto de la violación de sus derechos humanos, en los ámbitos público y privado, conformada por el conjunto de conductas misóginas que pueden conllevar impunidad social y del estado y puede culminar en homicidio y otras formas de muerte violenta de mujeres" (Diario Oficial de la Federación, 2007: 6).

\section{El espacio y el feminicidio}

El espacio feminicida se ha colocado como objeto de estudio de la geografía junto con otros conceptos que conforman la espacialidad de la violencia. En este estudio consideramos el concepto de espacio desde la tradición de Henri Lefebvre (1974), Milton Santos (2000) y Doreen Massey (2007), sin desconocer que existen tradiciones cartesianas como la de Schaefer (1971), fenomenológicas como la de Yi Fu Tuan (2001), o posmodernas como la de Joan Nogué (2014), además de otros referentes de la condición social del espacio — como territorio, lugar, paisaje, región — sus expresiones escalares y aquellas que implican las transformaciones de la segunda naturaleza. Recurrimos al concepto de espacio desde la geografía crítica para abordar el problema del feminicidio y evidenciar el alto contenido político — es decir, destacando la dimensión del poder-.

Lefebvre (2013) sostiene que “...el espacio es significante, ¿pero de qué? De lo que es preciso hacer y no hacer, y eso nos remite al poder. El mensaje del poder, no obstante, es siempre voluntariamente confuso, encubierto. El espacio del poder no dice todo, fundamentalmente dice lo prohibido. Su modo de existencia, su 'realidad' práctica (incluyendo su forma) difiere radicalmente de la realidad (de estar ahí) ..." (193194). El mismo autor identifica que "el espacio ordena los cuerpos, prescribe o proscribe los gestos, los trayectos y los recorridos" (Lefebvre, 2013: 194). Lefebvre reconoció que desde Vitruvio la forma se asocia a la función y ese criterio es un código equivalente a la legibilidad del espacio, pero 
La legibilidad aparente enmascara más de lo que declara; disimula precisamente lo que "es" visible-legible, sus trampas - lo que "es" la verticalidad - la arrogancia, voluntad de poder, exhibición de la virilidad militar y policial, la dimensión fálica, la analogía espacial de la brutalidad masculina. Nada puede darse por sentado en el espacio, porque se trata de actos reales o posibles y no de estados mentales, relatos mejores o peor contados. (Lefebvre, 2013: 195)

Lefebvre nos indica dos elementos fundamentales para este análisis: primero, que el espacio ordena a los cuerpos como un acto de represión y, segundo, que el espacio manifiesta una dimensión de poder masculino. El poder se aborda fundamentalmente desde el capitalismo, pero la producción del espacio no se limita a éste; también aparece el patriarcado. Sin embargo, la geografía feminista ha generado propuestas de análisis para evidenciar los espacios de represión y contribuye en la reflexión sobre las formas de mitigación y transformación de esta condición del patriarcado inscrito en el espacio. Desde la geografía feminista se ha propuesto la producción de un espacio libre de violencia.

Sin embargo, no es un problema menor la producción de un espacio libre de violencia, sino un proceso extremadamente complejo porque deben articularse todas las condiciones sociales que constituyen al espacio, como hasta ahora lo está el patriarcado, ensamblado en lo jurídico-político, lo social, cultural, lo científico-técnico y lo económico (Santos, 1986). Lefebvre identifica las representaciones del espacio como formas ideológicas y en la "historia del espacio [se] mostraría la génesis (y, por consiguiente, las condiciones en el tiempo) de esas realidades que algunos geógrafos designan como redes y que están subordinadas al armazón de la política" (Lefebvre, 2013: 170). Por ello, iniciaremos la crítica a los espacios del feminicidio con las transformaciones jurídicas en el contexto de la política, para mostrar la complejidad de una verdadera transformación espacial que no se agota en esta esfera jurídica, por muy importante que sea.

Doreen Massey (2007) y Milton Santos (2000) dejaron muy claro que las acciones importan, pero también las omisiones; Peter Taylor (1994: 33), desde la geografía política también habló del papel de no tomar decisiones para alcanzar ciertos fines. El espacio es una totalidad metodológica integrada por elementos sociales, jurídico-políticos, económicos, científico-técnicos que transforman la naturaleza originaria en una segunda naturaleza (Santos, 2000). En todos eso ámbitos las acciones y las omisiones vinculadas a la violencia hacia las mujeres están presentes en todas las escalas - local, estatal, nacional y global—, en todos los espacios — públicos, privados, virtuales y en los medios de comunicación masiva-, así como en los espacios de ocio, del entretenimiento y la cultura.

El cambio a una vida libre de violencia debe transformar la base de las relaciones sociales de producción y reproducción, sea capital y trabajo, subordinación de la mujer. No es suficiente con modificar leyes y el sistema jurídico; si bien son indispensables para posibilitar los cambios, es conveniente iniciar, en la complejidad de la transfor- 
mación espacial, la permuta de al menos uno de los niveles que la constituyen. ¿Qué es lo que realmente puede desterrar la violencia feminicida de nuestras sociedades? Primero tenemos que problematizar las violencias e identificar aquellas que se manifiestan en los cuerpos de las mujeres a través de golpes, mutilaciones, desapariciones y finalmente la muerte, pero también el acoso, menosprecio, invisibilización o presión psicológica y económica, entre otras. La naturalización de la violencia como algo "normalizado socialmente" ha sido la base de esta violencia. El cambio cultural es fundamental; se ha iniciado y lo identificamos con el feminismo en la tradición occidental desde el siglo XVIII. En los últimos cien años ha tenido una mayor presencia en el espacio público, con manifestaciones en la calle y con la implementación de políticas públicas, el debate en los espacios de representación política formal, e incluso en los medios de comunicación masiva. Esto ha permitido mostrar, a lo largo del desarrollo del feminismo, que no hay una sola violencia contra las mujeres, sino que ésta es múltiple y compleja. En el siglo XIX se identificaron las violencias asociadas a la raza (feminismo de la negritud), a la clase social (feminismo socialista, anarquista), y se han sumado las de orientación sexual, origen étnico, edad, condición migratoria y condición de discapacidad, entre otras formas sujetas a opresión.

Por ello, la erradicación de la violencia es un proceso de producción socioespacial que va más allá de construir refugios para las mujeres acosadas, colocar más luminarias en los caminos o videocámaras en los edificios y en el transporte; ésta es sólo una mirada cartesiana del espacio. Nosotras sostenemos que es necesario producir un espacio diferente en el que todos los niveles de la vida humana, de manera simultánea, coloquen en un lugar diferente y mejor a las mujeres, a todas las mujeres, a todo lo que las mujeres hacen y significan, sean transgénero, niñas, jóvenes, maduras o mayores, indígenas, afrodescendientes, mestizas, sin olvidar a las mujeres con discapacidad e independientemente de su estatus migratorio. El cambio cultural debe integrar transformaciones en la socialización (religiosa, económica, cultural) y en la educación formal que imparte el estado al menos en dos sentidos, tanto en los contenidos como en la socialización del espacio escolar: primero, en los niveles de participación en el espacio público, en donde se ha invisibilizado a las mujeres, sus aportes históricos y su presencia en la ciencia, basados en contenidos dicotómicos, desiguales, inequitativos y estereotipados sobre los roles de mujeres y hombres. En segundo lugar, en la socialización de las relaciones de género, donde se reproduce la violencia contra las mujeres con la invisibilización, menosprecio, estigmatización y reproducción de comportamientos misóginos y de acoso sexual contra las profesoras, estudiantes y trabajadoras.

En la actualidad, los medios de comunicación masiva juegan un papel fundamental en la reproducción de formas de socialización subordinada de los géneros y de la violencia contra las mujeres. Esto se da por factores que van desde la emisión de contenidos rosas que reproducen estereotipos de mujeres — madres, esposas, monjas — , o bien en su antípoda — presas, putas y locas, parafraseando un título de Marcela Lagarde (2005a) - , hasta elementos como encabezados, lenguaje soez y violento hacia las 


\title{
$36 \square$ LA VIOLENCIA FEMINICIDA: ABORDAJE DESDE UNA GEOGRAFÍA FEMINISTA
}

mujeres, así como con la presentación de los casos de feminicidios que juzgan y reproducen los estereotipos casi siempre responsabilizando a la víctima — es decir, la revictimizan - El poder investiga el feminicidio a través de instancias del estado, de los medios de comunicación o de las iglesias, quienes refuerzan el orden patriarcal y la cultura de la violencia al coludirse, naturalizarla y menospreciar a las mujeres, o tratan los crímenes como problemas personales, con detalles morbosos que atentan contra la dignidad de la mujer aun estando muerta.

Hasta aquí, mostramos algunos elementos que deben considerarse para la producción del espacio libre de violencia, a partir de un cambio profundo y radical de nuestra sociedad. A continuación, abordaremos algunos de los elementos fundamentales sobre los que se ha trabajado desde grupos feministas en términos de las bases legales y la política pública, que no han logrado aún abarcar todo el trabajo necesario para producir espacios seguros. Existen retos y resistencias en la producción del espacio libre de violencia, al que se ha definido como:

\begin{abstract}
inacabado, en proceso paulatino en el que intervienen distintos actores sociales y políticos en varios ámbitos [...] en los que se llevan a cabo acciones jurídicas, políticas, culturales, económicas, ideológicas y sociales, las que impulsadas desde el feminismo, presionan al estado para que las mujeres cuenten con el derecho a una vida libre de violencia, y con base en este conjunto de acciones cambien sus condiciones de vida, cuenten con acceso a la justicia a través de un marco jurídico con base en los derechos humanos de las mujeres, con políticas públicas las consideren sujetas de atención, donde las mujeres sean ciudadanas y estén empoderadas. En síntesis, se requieren medios económicos, jurídicos, ideológicos y psicológicos para vivir una vida libre de violencia y dejar de ser víctimas. (Damián, 2016: 35)
\end{abstract}

Nos centraremos en el ámbito jurídico, ante las dificultades que representan la identificación, nombrar, establecer la normatividad, la aplicación de políticas públicas y la sanción de conductas violentas contra las mujeres. Estas dificultades muestran las resistencias masculinas hacia la transformación del ámbito jurídico que constituye al espacio patriarcal. La tarea para evidenciar esta dominación no ha sido fácil; lleva siglos desarrollándose la demanda para que las mujeres tengamos los mismos derechos que los hombres y libres de violencia de género. Esto muestra que los cambios no surgirán sólo de la superestructura.

\section{La denuncia de la violencia contra las mujeres en distintas latitudes}

La violencia contra las mujeres en México es un problema de gran magnitud. Ante ello, el movimiento feminista ha sido un actor muy importante en su denuncia y en la exigencia de justicia. Fueron los familiares, principalmente las madres de las mujeres asesinadas, las activistas y las académicas del Colegio de la Frontera Norte quienes desde 1993 denunciaron la gran cantidad de mujeres que estaban siendo asesinadas en 
Ciudad Juárez, Chihuahua, cuando las instituciones de procuración de justicia no tenían el registro de dichos asesinatos y antes de que el concepto de feminicidio se planteara desde la academia, y con esto se abriera el debate nacional para su incorporación en los códigos penales. Ya existían otros tipos de violencias contra las mujeres nombrados así por las feministas, como en 1999, el caso contra Paulina, una niña de 13 años: después de una violación sexual, padeció la dilación de médicos, enfermeras, la intervención de la jerarquía católica y grupos como Provida presionando para evitar la interrupción del embarazo. La legislación local impidió la interrupción de éste al rebasar las cuatro semanas de gestación y poner en riesgo su vida, lo que constituyó la negación para decidir sobre su maternidad y su cuerpo. Este caso emblemático fue denunciado primero por las feministas de Mexicali y después fue discutido por las feministas de la Ciudad de México (Monárrez, 2000).

En 2001 el movimiento feminista en distintas ciudades se unió a la denuncia de los crímenes contra mujeres en Ciudad Juárez y se organizaron colectivos como Nuestras hijas de regreso a casa, con el lema "Ni una Más"; sin embargo, las instituciones de procuración de justicia no tenían el registro de dichos crímenes. De manera simultánea se planteó el concepto de feminicidio desde la academia. Las feministas abrieron el debate nacional; con el acompañamiento y denuncias de estos hechos se desarrolló un proceso paulatino que obligó a que estos crímenes fueran tipificados en los Códigos Penales Federal y Estatales como feminicidio.

Cabe destacar que en México las feministas han luchado por el derecho a la participación política y para decidir sobre su cuerpo. En el siglo xx hubo dos momentos emblemáticos de las manifestaciones feministas. Hace cien años las mujeres trabajadoras feministas de Yucatán demandaron el derecho a decidir sobre su cuerpo y lucharon por el aborto. En la década de 1970 las feministas de los Grupos Factor X y Alaide Foppa organizaron a las trabajadoras de la maquila en Tijuana y Mexicali, respectivamente, en contra de las precarias condiciones en las que laboraban y del acoso dentro y fuera de sus centros de trabajo.

\section{Feminicidio}

El posicionamiento del pensamiento feminista se ha nutrido con reflexiones y discusiones desde hace más de cuatro décadas (Carosio, 2009). Las feministas académicas han investigado, nombrado e identificado los distintos tipos de violencia -intrafamiliar, contra mujeres embarazadas, violencia sexual, entre otras - debido al ejercicio de poder y de lugares, formas y grados de opresión implicadas (Doan, 2010). La violencia extrema y los crímenes contra las mujeres se conceptualizaron como feminicidio (Lagarde, 2005a; Russel y Radford, 2006) visibilizándose en México en la frontera norte (Monárrez, 2000). De acuerdo con Moscoso Urzúa, Pérez Garrido y Estrada (2012), la primera aproximación al tema la realizó Diana Russell hacia 1976 cuando impulsó el término femicidio ante el Tribunal Internacional sobre Crímenes contra Mujeres en 
Bruselas para referirse a la violencia extrema ejercida contra las mujeres. En 1990, junto con Jane Caputi, definió el asesinato de mujeres por hombres motivado por el odio, desprecio, placer o sentido de propiedad de las mujeres. Marcela Lagarde (2008) define el feminicidio como genocidio contra mujeres que ocurre cuando las condiciones históricas generan prácticas sociales que permiten atentados violentos contra la integridad, la salud, las libertades y la vida de niñas y mujeres. En el feminicidio concurren, en tiempo y espacio, daños contra niñas y mujeres realizados por conocidos y desconocidos, por violentos - en ocasiones violadores - y asesinos individuales y grupales, ocasionales o profesionales, que conducen a la muerte cruel de las víctimas.

En las últimas décadas, en América Latina la tipificación del delito de feminicidio ha llevado a importantes movilizaciones para su reconocimiento y penalización tipificada. Existe una fuerte presión del trabajo feminista para que los crímenes contra las mujeres se consignen, investiguen, sancionen y se juzguen como feminicidios y, en consecuencia, se consoliden instituciones y procedimientos para su atracción, atención $\mathrm{y}$ acceso a la justicia.

En Guatemala, Perú y México las organizaciones de mujeres han asumido, en casos específicos, el rol de sujeto activo procesal (querellante adhesivo o acusador coadyuvante), hecho que le ha permitido actuar dentro del proceso penal junto al órgano acusador y consolidar la acusación penal: proponer y rechazar pruebas, solicitar la incorporación de métodos especiales de investigación, así como señalar y acusar a los funcionarios que obstaculizaron el acceso a la justicia de las mujeres. (Garita, 2014: 12)

Aunque en México la inclusión del delito de feminicidio en los códigos penales estatales se inició en 2010 en el Estado de Guerrero y concluyó en 2017 en el Estado de Chihuahua, todavía es un proceso inacabado que requiere la armonización del Código Penal Federal con los estatales para que se tengan los mismos criterios de investigación, sanción y aplicación de la justicia. El hecho de que en los códigos penales se tome en cuenta que en los crímenes contra las mujeres existió una relación de poder es un avance importante (Riquer y Castro, 2012). Sin embargo, los procesos de procuración e impartición de justica son lentos y las resistencias para juzgar con una perspectiva de género son muy complicadas en México debido a la cultura patriarcal en la que impartidoras e impartidores de justicia fueron formados, lo que ocasiona juicios sin la consideración de género existentes en el marco jurídico.

\section{La violencia feminicida en Ciudad Juárez: el caso paradigmático}

Ciudad Juárez, Chihuahua, es el espacio paradigmático en México en el que se identificó en 1993 por primera vez el feminicidio. La investigadora Julia Monárrez documentó con antelación los crímenes cometidos contra mujeres; sin embargo, en ese año 
se inició el registro de los hechos. Monárrez propuso la categoría de feminicidio sexual sistémico entendido como

el asesinato codificado de niñas y mujeres por ser mujeres cuyos cuerpos expropiados han sido torturados, violados, asesinados y arrojados en escenarios transgresivos por hombres que hacen uso de la misoginia y el sexismo para delinear cruelmente las fronteras de género por medio de un terrorismo de estado secundado por los grupos hegemónicos que refuerzan el dominio masculino y sujeta a familiares de víctimas y a todas las mujeres a una inseguridad crónica y profunda a través de un período continuo e ilimitado de impunidad y complicidades. (Monárrez, 2004: 9)

Por su parte, la geógrafa Melisa Wright caracterizó la lucha por la justicia y la erradicación de la violencia contra las mujeres en Ciudad Juárez con la manifestación de dos actores importantes: las familiares de las víctimas y los grupos de mujeres activistas (Wright, 2013). Además, en 2002 se conformaron distintas instituciones como el Instituto Chihuahuense de las Mujeres; sin embargo, la Fiscalía Especial para la Investigación de Homicidios contra Mujeres (FEIHM) no satisfizo las expectativas de las luchadoras sociales, porque las titulares de este órgano respondían a otros objetivos políticos y carecían de perspectiva de género.

Marcela Lagarde (2005a) refiere la concurrencia de manera criminal en el feminicidio del silencio, la omisión, la negligencia y la colusión de autoridades encargadas de prevenir y erradicar estos crímenes. Hay feminicidio cuando el estado no otorga garantías a las mujeres y no crea las condiciones de seguridad para sus vidas en la comunidad, en la casa, en los espacios de trabajo, de tránsito o de esparcimiento. El feminicidio se reproduce cuando las autoridades no realizan con eficiencia sus funciones. Si el estado falla se crea impunidad, la delincuencia prolifera y el feminicidio no llega a su fin. Por eso, el feminicidio es un crimen de estado. Si no se previene, si no se atiende, si no se investiga y si no se sanciona, se incita.

La falta de la procuración de justicia por parte de los tres órdenes de gobierno en Ciudad Juárez (municipal, estatal y federal) es opresora. Familiares de tres víctimas encontradas sin vida en un campo algodonero de Ciudad Juárez el 6 de noviembre de 2001 acudieron a la Corte Interamericana de Derechos Humanos para denunciar los hechos. En noviembre de 2009 la Corte Interamericana concluyó que

desde que se denunció la desaparición de las tres víctimas hasta que se encontraron los cuerpos, las autoridades competentes sólo registraron las desapariciones, solicitaron a la Policía Judicial que investigara, emitieron un oficio del Programa de Atención a Víctimas de los Delitos, elaboraron carteles indicando que las víctimas habían desaparecido y recibieron testimonios de algunas personas. Asimismo, la Corte IDH constató que la actitud de las autoridades, al minimizar la desaparición de las jóvenes con comentarios discriminatorios en razón de su género y edad, hacía presumir que las autoridades eran indiferentes a las denuncias de los familiares y que no investigaron diligentemente las desapariciones a efecto de prevenir daños a la integridad psíquica o física y/o la muerte de las jóvenes, la corte condenó al gobierno de México por 
haber sido omiso y no garantizar seguridad a las mujeres. (Corte Interamericana de Derechos Humanos, 2009).

En febrero de 2008, representantes de las familias de las víctimas, la Asociación Nacional de Abogados Democráticos A. C., el Comité de América Latina y el Caribe para la Defensa de los Derechos de la Mujer, la Red Ciudadana de No Violencia y por la Dignidad Humana y Centro para el Desarrollo Integral de la Mujer A. C. exigieron a la corte investigar once feminicidios más. La falta de investigación pericial profesional incurre en graves omisiones; ante esta situación, abogadas, investigadoras y feministas presionaron para que los crímenes no quedaran en la impunidad (Corte Interamericana de Derechos Humanos, 2009; Vázquez, 2011).

En 2005, la Comisión Especial para Conocer y Dar Seguimiento a las Investigaciones Relacionadas con los Feminicidios en la República Mexicana y a la Procuración de Justicia Vinculada (Comisión Especial de Feminicidios), constituida por la LIX Legislatura de la Cámara de Diputados de México, desarrolló una investigación a escala nacional con la participación de feministas, dando cuenta del problema a escala nacional, estatal y municipal (Damián, 2010). Se discutieron las necesarias acciones coordinadas para la prevención de la violencia, el monitoreo y relatoría. Se presentó una extensa demanda para esclarecer los feminicidios en Chihuahua. La discusión del feminicidio como categoría de análisis se incluyó en los debates (LIX Legislatura, 2005) y el papel de las feministas también fue central para denunciar los crímenes y dar seguimiento a las acciones de justicia y la prevención.

La geografía feminista se interesa en comprender las manifestaciones de la violencia contra las mujeres y cómo se produce este espacio de violencia, dónde y cuándo se presentan estos crímenes, utilizando la cartografía para su registro, memoria y denuncia (Damián, 2010; 2012). Consideramos central para frenar la violencia feminicida el enfoque respecto a la voluntad política, cambios culturales y normatividad jurídica que impida la impunidad; todos ellos, actuando de manera interconectada, reducirán los espacios de violencia y propiciarán el respeto al cuerpo de las mujeres, a su dignidad y vida plena. En ese sentido contribuye el trabajo de georreferenciación, desmontando los mitos que se generan con base en estereotipos de las víctimas, de los lugares donde sus cuerpos fueron encontrados. Se realizó el seguimiento y mapeo de los perfiles sociales de los casos de feminicidio de Ciudad Juárez desde la década de 1990; entre los hallazgos se encontró que había víctimas niñas, adolescentes, jóvenes, maduras y ancianas, también mujeres amas de casa, estudiantes, meseras, solteras, casadas, divorciadas y embarazadas; no sólo eran víctimas las mujeres trabajadoras de las maquiladoras, como se pensaba a inicios de 1990. "De 1993 a 2005, el $23 \%$ de las víctimas se localizaron en sus casas, el $7 \%$ en negocios, el $32 \%$ en la vía pública, el $23 \%$ en caminos y carreteras, el $15 \%$ en lotes baldíos" (citado en Damián, 2010: 100). Los resultados y mapas pueden consultarse en los informes de la citada Comisión Especial de Feminicidio (LIX Legislatura, 2005). 


\section{La investigación}

La Comisión Especial de Feminicidios publicó la primera investigación diagnóstica, en la que participaron investigadoras de distintas universidades del país. Sólo en 2004 más de 1205 mujeres fueron asesinadas en México y documentaron los distintos tipos de violencia contra las mujeres en las 32 entidades federativas; la investigación utilizó cartografía feminista y respaldó la elaboración de la Ley General de Acceso de las Mujeres a una Vida Libre de Violencia, propuesta aprobada por el Poder Legislativo. Esta ley identifica los distintos tipos de violencia, los lugares donde se presenta, y establece mecanismos de prevención, atención, sanción y erradicación. Involucra las tres escalas de gobierno y atiende la alerta de violencia de género contra las mujeres, solicitada por las organizaciones feministas. Este mecanismo pretende la implementación de medidas extraordinarias en aquellos lugares con la presencia constante y sistemática de la violencia feminicida. Es un primer intento de espacializar las políticas de atención para disminuir y evitar el incremento de la violencia contra las mujeres en la escala estatal y municipal.

\section{Elaboración de marcos jurídicos para una vida libre de violencia para las mujeres y tipificación del delito de feminicidio}

En México se cuenta con la Ley General de Igualdad entre Mujeres y Hombres (LGIMH) y con la Ley General de Acceso de las Mujeres a una Vida Libre de Violencia (LGAmvLV, en adelante Ley de Acceso a una Vida Libre de Violencia), vigentes desde 2006 y 2007, respectivamente, gracias al trabajo de las legisladoras feministas de la LIX Legislatura de la Cámara de Diputados. Estas leyes son de vital importancia porque colocan a las mujeres como sujetas de derecho; tienen como principios la igualdad, la no discriminación y la equidad. Ambas leyes, al ser generales, tienen como objetivo la coordinación entre la federación, las entidades federativas y los municipios, para prevenir la discriminación en contra de las mujeres. Por una parte, la Ley de Acceso a una Vida Libre de Violencia busca la prevención, atención, sanción y erradicación de la violencia contra ellas, así como establecer los principios y modalidades para garantizar la vida libre de violencia y la implementación del mecanismo de Alerta de Violencia de Género, la que también puede ser solicitada por organizaciones de derechos humanos. Respecto a las sanciones por delitos cometidos, estas se incluyeron en los códigos penales y de procedimiento.

La discusión conceptual del feminicidio, desde una geografía feminista, se sitúa espacial y temporalmente para explicarlo en su complejidad considerando todos los aportes académicos para identificar las regularidades y patrones del feminicidio. El feminismo traspasa las aulas e instalaciones universitarias para entender los impactos de las tomas de decisión en ciertos lugares, espacios de lo cotidiano. Esto implica el 
reconocimiento de las relaciones de poder y discriminación de la cultura misógina en el hogar, la escuela, el trabajo, la ciudad y el medio rural. A través de las políticas públicas deben reforzarse las decisiones concretas de los diversos órdenes de gobierno para aplicar las sanciones, el acceso a la justicia y la investigación de los crímenes contra las mujeres desde una perspectiva de género. Las discusiones conceptuales y jurisdiccionales en torno al feminicidio identifican que el estado es responsable de no actuar en tiempo y forma (Lagarde, 2005b: 150). Respecto a la tipificación del delito, el bien protegido que se vulnera es la vida de las mujeres. Desde el feminismo estos crímenes se consideren feminicidios y no homicidios o, peor aún, suicidios; por ello, es crucial que los peritajes de las instituciones de la procuración de justicia se sustenten en los protocolos de actuación específicos e identifiquen los crímenes cometidos por razones de género. En el código Penal de la Federación de 2012, Artículo 325, se considera el delito de feminicidio cuando:

I. La víctima presente signos de violencia sexual de cualquier tipo.

II. A la víctima se le hayan infligido lesiones o mutilaciones infamantes o degradantes, previas o posteriores a la privación de la vida o actos de necrofilia.

III. Existan antecedentes o datos de cualquier tipo de violencia en el ámbito familiar, laboral o escolar, del sujeto activo en contra de la víctima.

IV. Haya existido entre el activo y la víctima una relación sentimental, afectiva o de confianza. (Cámara de Diputados, 2014)

El proceso de tipificación del delito de feminicidio en México ha sido tardado, considerando que en 2010 comenzaron los cambios en los marcos jurídicos. La primera entidad en hacerlo fue Guerrero. En 2011 procedió la tipificación en los estados de Estado de México, Guanajuato, Morelos, Colima, Durango, San Luis Potosí, Tamaulipas, Veracruz y la Ciudad de México. En 2012, lo tipificaron los estados de Baja California, Campeche, Coahuila, Chiapas, Jalisco, Nayarit, Oaxaca, Puebla, Quintana Roo, Sinaloa, Tabasco, Yucatán, Tlaxcala y Zacatecas. En 2013, Aguascalientes, Nuevo León, Hidalgo y Sonora. En 2014, Baja California Sur y Michoacán; y en 2017, Chihuahua, donde, paradójicamente, se dieron a conocer los primeros casos de feminicidio (véase mapa 1 ).

La producción del espacio libre de violencia para las mujeres se logrará en la medida en que haya acceso a la justicia y se erradiquen los hechos de violencia. Para ello, el primer recurso es el reconocimiento legal de este problema público; otro recurso, aún más difícil porque significa vencer muchos obstáculos, es la procuración e impartición de justicia, la cual consta de varias etapas: primero, la investigación pericial es de suma importancia; responde a los tipos penales. Otro momento son los juicios de los casos y, finalmente, la resolución para que el delito no quede en la impunidad y la reparación del daño. Todos son cruciales; sin embargo, investigaciones recientes en materia de procuración de justicia en México señalan que en 2016 solamente un 3.39 \% de todos los casos de muerte de niñas y mujeres se reportaron como feminicidio ( 84 


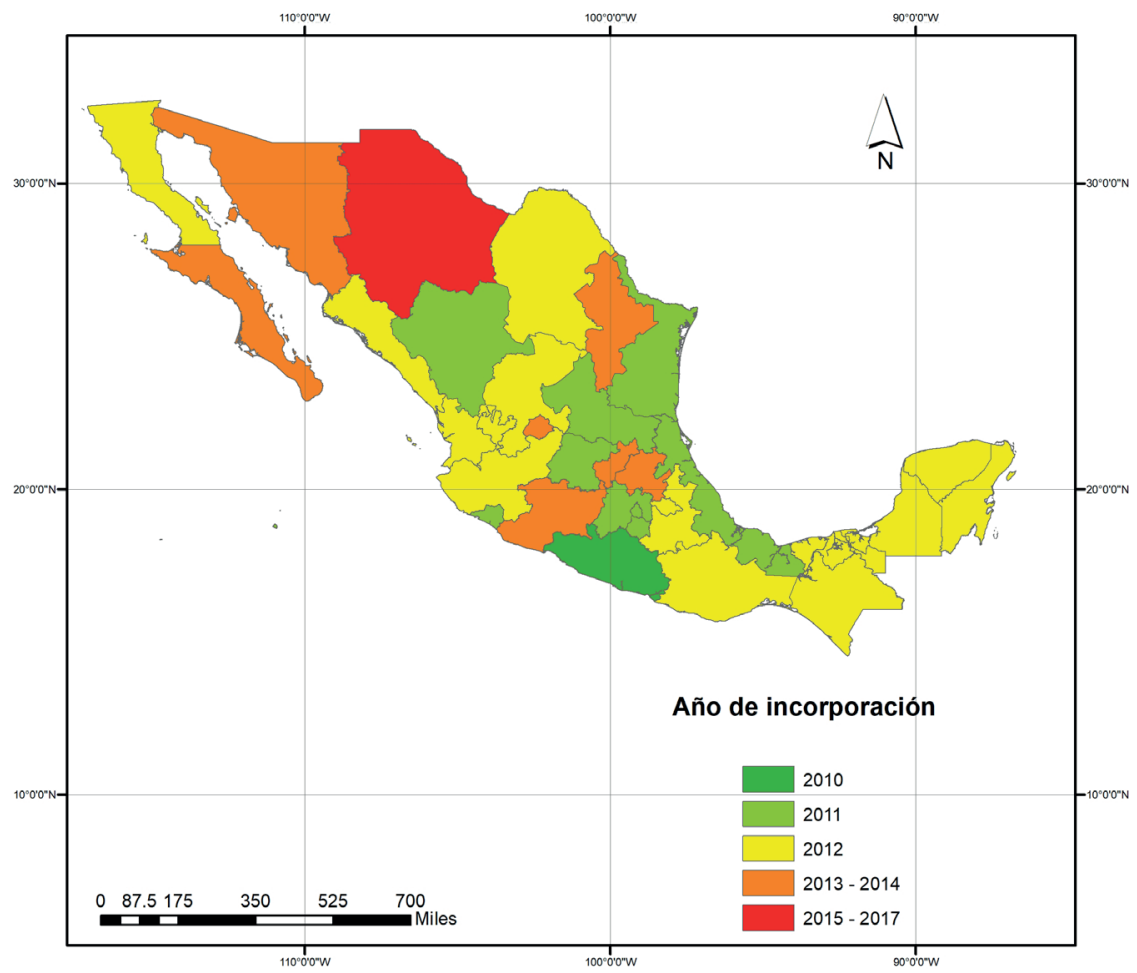

Mapa 1 Año de incorporación del tipo penal feminicidio, 2010-2017

Fuente: elaboración propia con base en la Comision Nacional de Derechos Humanos. 2019

Recomendación General Número 40 Sobre violencia feminicida y el derecho a las mujeres a una vida libre de violencia.

casos). Asimismo, destaca que el $69.43 \%$ fueron clasificados como homicidio culposo, mientras que un $25.61 \%$ como homicidio doloso (Cruzvillegas Fuentes, s. f.: 6). En el año 2018, todas las entidades ya habían incorporado el tipo penal de feminicidio, aunque algunas sólo lo consideran como agravantes.

\section{Procuración de justicia}

Las procuradurías de justicia se encargan de las averiguaciones previas (procedimiento anterior al proceso penal), que se lleva a cabo por un órgano del Poder Ejecutivo, ya sea federal o local. Las averiguaciones se inician a partir de que el agente del Ministerio Público, como autoridad investigadora, tiene conocimiento mediante una denuncia o querella de hechos que pueden constituir un delito (Poder Judicial de la Ciudad de México, 2016: 431). El Censo Nacional de Procuración de Justicia Estatal da segui- 
miento a la gestión y desempeño de las instituciones que integran a la Procuraduría General de Justicia o Fiscalía General de cada entidad federativa, específicamente las funciones son del gobierno (INEGI, 2019). Dicho Censo fue consultado para dar seguimiento a los registros correspondientes a 2018 e indica que a escala nacional hubo 925 casos de feminicidio consumados ${ }^{1}$ y en grado de tentativa ${ }^{2}$ en procesos iniciados (INEGI, 2019).

En el mapa 2 se muestran las entidades por número de delitos de feminicidio consumados y grado de tentativa que están siendo investigados por las procuradurías de justicia estatales. Baja California Sur no registró ninguno; esto responde a que no cuenta con este delito. La denominación del tipo penal más semejante al feminicidio en esta entidad es "homicidio agravado por feminicidio". Sin embargo, nueve entidades -Quintana Roo, Yucatán, Campeche, Tlaxcala, Querétaro, Aguascalientes, Nayarit, Durango y Tamaulipas - son las que registraron de uno a trece casos consumados y en grado de tentativa. Ocho entidades - Baja California, Coahuila, San Luis Potosí, Zacatecas, Guanajuato, Michoacán, Colima e Hidalgo - estuvieron en el segundo rango de catorce a veintiocho casos. Seis entidades estuvieron en el rango de veintinueve a cuarenta casos: Chiapas, Tabasco, Oaxaca, Guerrero, Puebla, Jalisco. Tres entidades estuvieron en el rango de cuarenta y uno a cincuenta y dos casos: Sinaloa, Chihuahua y Sonora. Tres entidades estuvieron en el rango de cincuenta y dos a ciento nueve casos: Estado de México, Veracruz y Nuevo León.

Sin embargo, los registros de homicidio contra las mujeres muestran valores más elevados que los de feminicidio, particularmente en los estados de Baja California, Guanajuato, México y Chihuahua. En general, en las Procuradurías de justicia estatales, presumiblemente existe una tendencia de subregistro de los feminicidios por la escasa investigación realizada (véase Tabla 1). Desde la procuración de justicia se argumenta que una de las razones para no caracterizar los crímenes contra mujeres como feminicidios se debe a que "faltan las razones de género". ${ }^{3}$

Si el delito de feminicidio se contrasta con el de homicidios contra mujeres, se podrán notar diferencias en las sentencias y en las penalidades; en consecuencia, al tipificarlo como homicidio se diluye el entramado de poder patriarcal que da cabida al feminicidio. Por lo tanto, la investigación y posterior juicio penal desde la perspectiva de género significa el reconocimiento del feminicidio para visibilizar los contextos de poder y de desigualdad en los que se encontraban las víctimas.

\footnotetext{
${ }^{1}$ Significa que los actos reclamados ya han surtido todos sus efectos pero, jurídica y materialmente, éstos pueden ser revertidos mediante la sentencia que concede el amparo; es procedente el juicio de garantías (Montoya Pérez, 2016).

${ }^{2}$ Significa que el delito no se concretó por un factor externo.

${ }^{3}$ Solo 16 entidades establecen "razones de género" para constituir el tipo penal: Baja California, Campeche, Chiapas, Colima, Ciudad de México, Estado de México, Hidalgo, Jalisco, Morelos, Quintana Roo, Sinaloa, Tabasco, Tamaulipas, Veracruz, Yucatán y Zacatecas). Esto motiva a tipificar el feminicidio tiene que ver con combatir la invisibilidad, combatir la discriminación de jure y de facto, combatir la impunidad y combatir la permisibilidad social (Hernández Ramírez, 2014).
} 


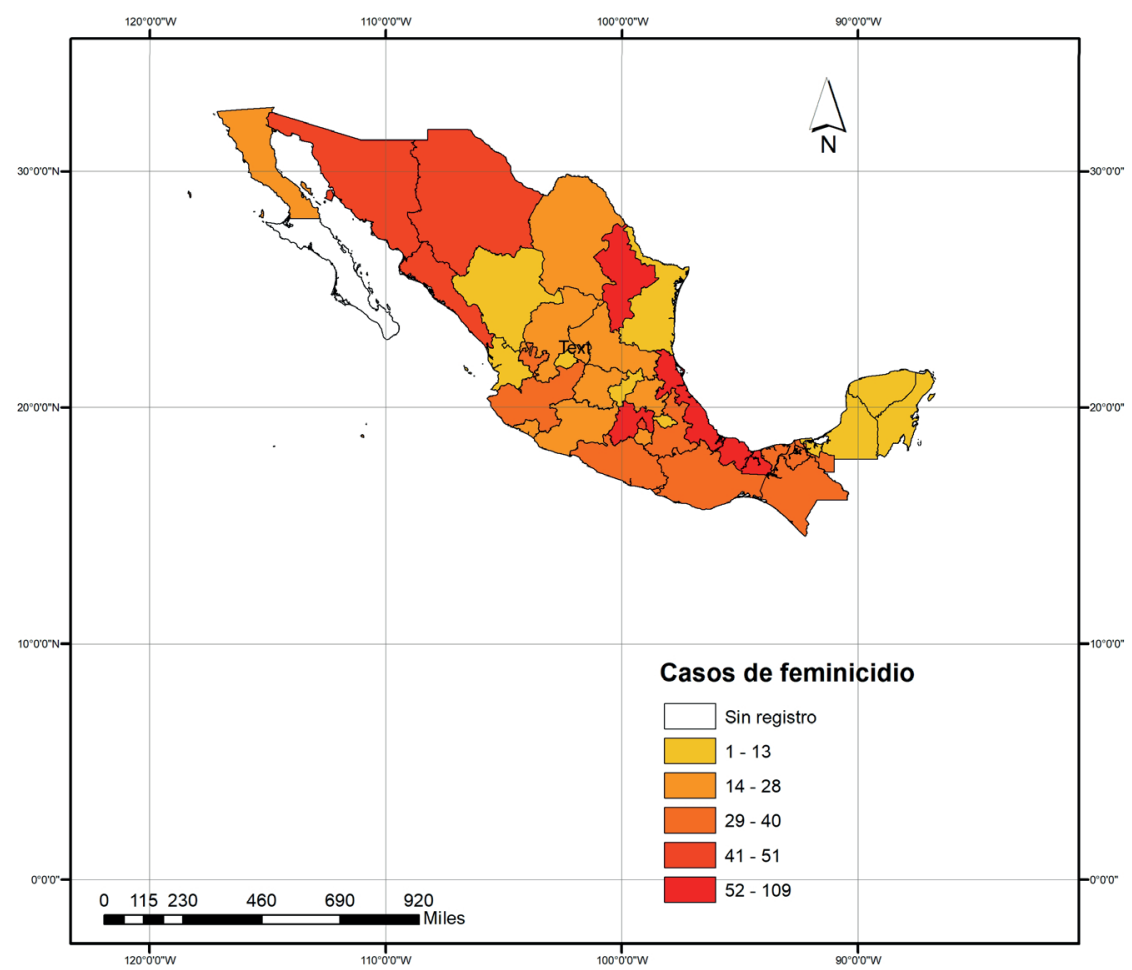

Mapa 2 Delito de feminicidio y en grado de tentativa. Procuración de Justicia. 2018 Fuente: elaboración propia a partir del Instituto Nacional de Estadística Geografía e Informática Censo Nacional de Procuración de Justicia Estatal. 2019

La tabla 1 muestra que la violencia contra las mujeres se investiga bajo el supuesto de homicidio en mayor proporción en comparación al delito de feminicidio. Como puede verse, no hay punto de comparación entre uno y otro, lo que demuestra que, a pesar de contar con un marco jurídico en contra de la violencia feminicida, éste no parece aplicarse. Para comprobar que fueron casos de feminicidio deben ser considerados todos elementos periciales, circunstanciales y lugares (casi todos son pasados por alto). Los códigos penales de las entidades difieren en cómo definen los delitos; sus criterios varían en los agravantes y atenuantes. ${ }^{4}$

4 “Baja California, Coahuila, Michoacán, Querétaro, San Luis Potosí y Zacatecas tienen como pena mínima de prisión 20 años. Sin embargo, otras entidades como Aguascalientes, Campeche, Chiapas, Colima, Durango, Estado de México, Guerrero, Jalisco, Morelos, Nuevo León, Puebla, Tabasco, Tamaulipas, Tlaxcala y Veracruz instauraron penas mínimas de 40 años de prisión y destaca Oaxaca que la mínima es de 50 años" (Comisión Nacional de Derechos Humanos, 2019: 26). "La 
$46 \square$ LA VIOLENCIA FEMINICIDA: ABORDAJE DESDE UNA GEOGRAFÍA FEMINISTA

Tabla 1

Número de homicidios contra mujeres por entidad federativa en investigación de la Procuración de Justicia, 2018

\begin{tabular}{|c|c|c|}
\hline & Homicidio contra mujeres & Feminicidio \\
\hline Estados Unidos Mexicanos & 7286 & 842 \\
\hline Aguascalientes & 84 & 4 \\
\hline Baja California & 1652 & 26 \\
\hline Baja California Sur & 40 & 0 \\
\hline Campeche & 33 & 8 \\
\hline Coahuila de Zaragoza & 102 & 16 \\
\hline Colima & 0 & 0 \\
\hline Chiapas & 214 & 37 \\
\hline Chihuahua & 433 & 54 \\
\hline Ciudad de México & 328 & 48 \\
\hline Durango & 40 & 6 \\
\hline Guanajuato & 618 & 21 \\
\hline Guerrero & 351 & 33 \\
\hline Hidalgo & 157 & 20 \\
\hline Jalisco & 392 & 33 \\
\hline México & 440 & 112 \\
\hline Michoacán de Ocampo & 445 & 24 \\
\hline Morelos & 190 & 28 \\
\hline Nayarit & 70 & 6 \\
\hline Nuevo León & 120 & 79 \\
\hline Oaxaca & 237 & 29 \\
\hline Puebla & 309 & 35 \\
\hline Querétaro & 71 & 7 \\
\hline Quintana Roo & 106 & 8 \\
\hline San Luis Potosí & 91 & 27 \\
\hline Sinaloa & 133 & 50 \\
\hline Sonora & 133 & 48 \\
\hline Tabasco & 83 & 40 \\
\hline Tamaulipas & 199 & 13 \\
\hline Tlaxcala & 65 & 3 \\
\hline Veracruz de Ignacio de la Llave & 0 & 108 \\
\hline Yucatán & 1 & 7 \\
\hline Zacatecas & 149 & 20 \\
\hline
\end{tabular}

Fuente: Elaboración propia a partir de INEGI (2019) 
El problema de la violencia feminicida tiene que entenderse más allá de las penas condenatorias, orientado hacia el acceso a la justicia y la aplicación irrestricta de la Ley para evitar los hechos que acaban con la vida de las mujeres. Los feminicidas están libres por la falta de profesionalismo en las investigaciones periciales, la escasa voluntad política de servidores y servidoras públicas de procuración de justicia, la falta de ética y la corrupción de las y los impartidores de justicia que manipulan las decisiones. Esto en sí mismo puede tipificarse como delito de encubrimiento. Por otro lado, una vez que el Ministerio Público integra las averiguaciones previas y determina que hay delito que perseguir, sigue el turno del poder judicial, quien se encargará de juzgar los casos y dictar sentencia. No obstante, cabe el riesgo de que contradiga a la procuraduría de justicia y concluya que no hay elementos suficientes para la acción penal.

\section{Impartición de justicia}

Al poder judicial le compete la impartición de justicia, interpretación de las leyes, vigilar su cumplimiento, resolver conflictos entre las personas y entre los órganos del poder público, así como proteger los Derechos Humanos establecidos en la Constitución Federal y los tratados internacionales, además de los derechos político-electorales de las personas (Poder Judicial de la Federación, 2009).

En el mapa 3 se puede ver que el número de casos juzgados como feminicidio disminuyen si se comparan con los reportados por el Censo de Procuración de Justicia, lo cual es sumamente alarmante porque las y los impartidores de justicia descartan los casos, o en varias ocasiones resuelven que se trataban de suicidios. Al revisar el Censo de Impartición de Justicia, se detectaron algunas entidades que no enviaron información al Instituto Nacional de Estadística, Geografía e Informática, entre las que se encuentran Estado de México, Baja California Sur, Nuevo León y Tamaulipas. Esto da cuenta del desinterés del poder judicial para dar un seguimiento puntual y serio a un problema que afecta a la mitad de su población; es una muestra más de la falta de voluntad política o incapacidad, lo que contribuye a producir espacios de violencia para las mujeres.

Es un reto enorme luchar para alcanzar la impartición de justicia con una perspectiva de género. Se requiere capacidad para cambiar las relaciones de poder inmersas en los crímenes contra las mujeres. Como puede verse, el problema del feminicidio entraña la falta de acceso a la justicia, a la reparación del daño a las y los familiares de las víctimas. 


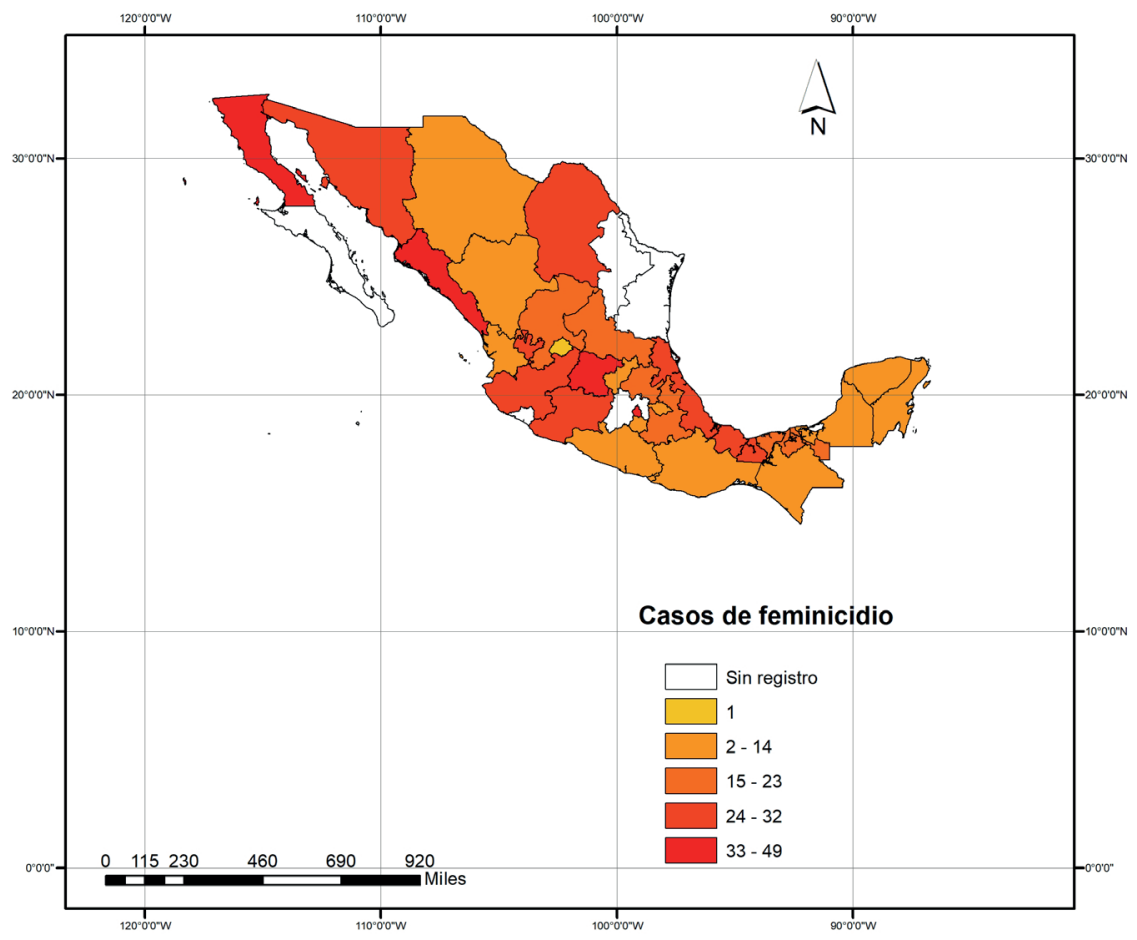

Mapa 3 Delitos cometidos a las víctimas por feminicidio. Impartición de Justicia. 2018 Fuente: elaboración propia a partir del Instituto Nacional de Estadística Geografía e Informática Censo de Impartición de Justicia Estatal

Consulta de delitos cometidos a las víctimas por entidad según cantidad. 2019

\section{Conclusiones}

La violencia contra las mujeres es un proceso social muy arraigado. Se ha demostrado que los movimientos feministas integrados por activistas, académicas y las mujeres desde la política formal y popular han contribuido a colocar el debate del feminicidio en el espacio público y en la deliberación política y a obtener el reconocimiento del problema, la redacción y aprobación de leyes y marcos jurídicos para sancionar, pensando en prevenir la violencia contra las mujeres. Los cambios en el marco jurídico para sancionar y erradicar la violencia son resultado de las feministas, las y los activistas, los familiares de las víctimas de violencia feminicida, así como la academia feminista al brindar soporte teórico, metodológico, argumentativo y acompañamiento a la sociedad civil en su demanda al estado e instancia de gobierno para su intervención. 
Las alertas de violencia de género solicitadas por las organizaciones feministas también han sido un motor de cambio. La alerta se solicita por la falta de políticas públicas que garanticen a las mujeres una vida libre de violencia, porque el feminicidio ocurre en espacios determinados - el primero, en el Estado de México- Se construye el espacio feminicida allí donde hay resistencias para establecer y hacer valer la normatividad; donde la lentitud en la procuración de justicia se exacerba; donde las y los abogados, jueces, ministerios públicos, médicos legistas, magistrados formados en perspectiva de género no ejercen su función ante la Ley.

Actualmente la presencia del movimiento feminista es más contundente y actúa en todo el territorio nacional, en municipios rurales y urbanos, en espacios conservadores donde es delito decidir sobre su propio cuerpo, como Guanajuato o Jalisco, respondiendo con violencia en los cuerpos de las mujeres. Las mujeres víctimas de feminicidio fueron vulneradas, violentadas, agredidas, violadas; el que hoy no estén aquí, se debe a inacciones sociales, jurídicas y políticas que en conjunto produjeron los espacios feminicidas. Todos los crímenes contra mujeres pudieron evitarse por la acción u omisión del estado, la sociedad cómplice y misógina que ocultó, evadió, no respondió, ni se pronunció para que los hechos se sancionaran y detuvieran. El feminicidio responde a un enorme espacio de poder patriarcal; va más allá del acto en sí mismo.

Para producir el espacio libre de violencia es necesario involucrar con mayor contundencia a la sociedad en la educación formal libre de sexismo, inequidad, violencia, a la socialización de las problemáticas, con intervenciones abiertas y consensadas, desde las escuelas de nivel básico hasta el universitario impulsando las causas feministas para transformar las relaciones misóginas establecidas históricamente y reproducidas por todas las instituciones, incluyendo las educativas y los medios. Debe reconocerse todo el trabajo realizado por las familias de las víctimas, por los colectivos feministas a lo largo y ancho del país en todos los espacios públicos y privados, en todos los municipios y alcaldías, el acompañamiento de la academia feminista y el impulso y contribución en el debate público. La deliberación ha logrado transformaciones jurídicas de la mayor importancia; falta su correlato en la aplicación de este marco jurídico, con mujeres y hombres que conozcan, dominen y apliquen la perspectiva de género para implementar justicia, pero también para demostrar que no habrá impunidad a quien ejerza violencia contra las mujeres.

Por ello, vemos la necesidad de que el feminismo sea una propuesta teórico-metodológica integrada en los contenidos académicos de todos los niveles educativos, para contribuir en el respeto a las mujeres. Al mismo tiempo, los medios de comunicación masiva pueden contribuir en la producción del espacio libre de violencia difundiendo las acciones y omisiones del poder judicial que generan violencia feminicida. La geografía habla del espacio como producción social, desde el feminismo queremos cambiar la realidad del espacio feminicida a un espacio libre de violencia con transformaciones de fondo contra el sistema patriarcal y no gatopardismos. 


\section{Referencias bibliográficas}

Cámara de Diputados. (2014). Código Penal Federal. México: Diario Oficial de la Federación.

CArosio, Alba. (2009). "Feminismo latinoamericano: imperativo ético para la emancipación". En Alicia Girón (Coord.), Género y globalización. Buenos Aires: CLACSO. 229-252.

Comisión Nacional de Derechos Humanos. (2019). Recomendación General Número 40 Sobre violencia feminicida y el derecho de las mujeres a una vida libre de violencia en México. Ciudad de México. Recuperado el 8 de agosto de 2020 de: https://www.cndh.org.mx/documento/recomendacion-general-402019

Corte Interamericana de Derechos Humanos. (2009). "Caso González y otras ('Campo Algodonero') vs. México". Recuperado 29 de septiembre 2020 de https://www. corteidh.or.cr/docs/casos/articulos/seriec_205_esp.pdf

Cruzvillegas Fuentes, María Eréndira (Coord.). (s. f.). Diagnóstico Nacional de Acceso a la Justicia y Violencia Feminicida 2016. México: Comisión Nacional de Derechos Humanos. Universidad Autónoma Metropolitana; plantel Iztapalapa. Recuperado de https://igualdaddegenero.cndh.org.mx/Content/doc/Publicaciones/DAJVF.pdf

DAMIÁN, Angélica Lucía. (2010). La manifestación espacial de la violencia feminicida en Naucalpan. (Tesis de maestría en Geografía, Universidad Nacional Autónoma de México).

DAmiÁN, Angélica Lucía. (2012). "La manifestación espacial de la violencia feminicida en el Estado de México. El caso Naucalpan, 2000-2006". En Norma Baca Tavira y Graciela Vélez Bautista (Coords.), Violencia, género y la persistencia de la desigualdad en el Estado de México. Buenos Aires, Mnemosyne. 39-62.

Damián, Angélica Lucía. (2016). El proceso de producción de un espacio libre de violencia para las mujeres en Naucalpan. (Tesis doctoral en Geografía, Universidad Nacional Autónoma de México).

Diario Oficial De La Federación. (2007). Ley General de Acceso de las Mujeres a una Vida Libre de Violencia. México.

Doan, Petra L. (2010). "The Tyranny of Gendered Spaces-Reflections from beyond the Gender Dichotomy". Gender, Place \& Culture: A Journal of Feminist Geography, 17(5), 635-654. http://doi.org/10.1080/0966369X.2010.503121

Garita, Ana Isabel. (2014). La regulación del delito de feminicidio en América Latina y el Caribe. Ciudad de Panamá: Secretariado de las Naciones Unidas UNETE para poner fin a la Violencia contra las Mujeres.

Instituto Nacional de Estadística Geografía e Informática. (2019). Censo Nacional de Procuración de Justicia Estatal. Ciudad de México. Recuperado el 2 julio de 2020 el https://www.inegi.org.mx/sistemas/Olap/Proyectos/bd/censos/gobierno2019/CNPJE2019/ProcProcJust.asp

Lagarde, Marcela. (2005a). Los cautiverios de las Mujeres: madresposas, monjas, putas, presas y locas. (Tesis doctoral, Universidad Nacional Autónoma de México). 
Lagarde, Marcela. (2005b) "El feminicidio, delito contra la humanidad”. En Feminicidio, Justicia y Derecho. México D. F.: H. Congreso de la Unión, Cámara de Diputados, LIX Legislatura. 151-164.

LAGARDE, Marcela. (2008). “Antropología, feminismo y política, violencia feminicida y derechos humanos de las mujeres". En Margaret Bullen y Carmen Diez Mintegui (Coords.), Retos teóricos y nuevas prácticas. San Sebastián: Ankulegi. 209-240.

Lefebvre, Henri. (2013 [1974]). La producción del espacio, Madrid: Capitán Swing.

LiX Legislatura Cámara de Diputados. (2005). Feminicidio, Justicia y Derecho. Recuperado el 29 de septiembre de 2020 de http://archivos.diputados.gob.mx/ Comisiones/Especiales/Feminicidios/docts/FJyD-interiores-web.pdf

Massey, Doreen. (2007). Conferencia 1. Geometrías del poder y la conceptualización del espacio. Conferencia dictada en la Universidad Central de Venezuela, Caracas, el 17 de septiembre de 2007. Recuperado el 15 de septiembre de 2019 de https:// ecumenico.org/geometrias-del-poder-y-la-conceptualizacion-del-es/

MonÁRrez, Julia. (2000). "La cultura del feminicidio en Ciudad Juárez, 1993-1999”. Frontera Norte, 12(23), 87-117.

MonÁRrez, Julia. (2004). "Elementos de análisis de feminicidio sexual sistémico en Ciudad Juárez para su viabilidad jurídica". Ponencia presentada en el Seminario Internacional: Feminicidio, Derecho y Justicia. México, D. F.. Recuperado el 14 de julio de 2020 de http://mujeresdeguatemala.org/wp-content/uploads/2014/06/ Elementos-del-feminicidio-sexual-siste\%CC\%81mico.pdf

Montoya Pérez, Oscar. (2016). Diccionario Jurídico. Recuperado el 5 de agosto de 2020 de http:/diccionariojuridico.mx/

Moscoso Urzúa, Valeria; Pérez Garrido, Ana Yeli; Estrada, María de la Luz. (2012). Violencia contra las Mujeres en el Estado de México. Informe de Impacto Psicosocial del Feminicidio de Nadia Alejandra Muciño Márquez. México D. F.: Comisión Mexicana de Defensa y Promoción de los Derechos Humanos A. C.

NoguÉ, Joan (2014). "Sentido del lugar, paisaje y conflicto". Geopolítica(s), 5(2), 155-163. Poder Judicial de la Ciudad de MéXico. (2016). Glosario. Recuperado el 2 de agosto de 2020 de http://www.poderjudicialcdmx.gob.mx/estadistica/wpcontent/ uploads/Glosario.pdf.

Poder Judicial de la Federación. (2009). El poder judicial de la federación al alcance de los niños. Recuperado el 2 de julio de 2020 de https://www.scjn.gob.mx/ sites/default/files/material_didactico/2016-11/EL\%20PJF\%20AL\%20AL\%20 ALCANCE_0.pdf

RiQuER, Florinda; CASTRO, Roberto (Coords.). (2012). Estudio Nacional sobre las Fuentes, Orígenes y Factores que Producen y Reproducen la Violencia contra las Mujeres. Comisión Nacional para Prevenir y Erradicar la Violencia contra las Mujeres. Recuperado de http://www.conavim.gob.mx/work/models/ CONAVIM/Resource/103/1/images/1PresentacionResultadosEstudioNacion alsobrelasFuentesOrigenes.pdf

Russell, Diana; Radford, Jill. (2006). Feminicidio: La politica del asesinato. México: CEICH-UNAM. 
Santos, Milton. (1986). "Espacio y método". Geocrítica, (65). http://www.ub.edu/ geocrit/geo65.htm

Santos, Milton. (2000). La naturaleza del espacio. Técnica y tiempo, razón y emoción. Barcelona: Ariel.

Schaefer, Fred. (1971). Excepcionalismo en Geografia. Barcelona: Universidad de Barcelona Publicaciones.

TAYlor, Peter. (1994 [1985]). Geografía Política, economía-mundo, estado-nación y localidad. Madrid: Trama Editorial.

Tuan Yi Fu. (2001). Space and Place. The Perspective of Experience. Minneapolis: University of Minnesota Press.

VÁzquEz, Santiago José. (2011). “El caso 'campo algodonero’ ante la Corte Interamericana de Derechos Humanos". Anuario mexicano de derecho internacional, 11. 515-561.

Wright, Melisa. (2013). "Feminicide, Narcoviolence, and Gentrification in Ciudad Juárez: The Feminist Fight". Environment and Planning D: Society and Space, 31(5), 830-845. http://doi.org/10.1068/d17812 